\title{
Ibutilide for treatment of atrial fibrillation in the emergency department
}

\author{
O Vikłorsdottir, A Henriksdottir, D O Arnar
}

Emerg Med J 2006;23:133-134. doi: 10.1136/emj.2004.021394

The purpose of this study was to assess the efficacy and safety of ibutilide, a class III antiarrhythmic drug, for acute treatment of atrial fibrillation (AF) in the emergency department (ED) setting. A retrospective analysis was done reviewing all patients with $A F$ who received ibutilide in the $E D$ in a university hospital setting. A total of 22 patients received ibutilide. Another 24 patients who received rate control drugs only served as a control group. Of the 22 patients who received ibutilide, $14(64 \%)$ converted to sinus rhythm. The mean (SD) rate of AF was 137 (24) bpm and the mean QT interval immediately after conversion to sinus rhythm was 420 (28) ms. There were no complications. In the rate control group 7 patients (29\%) converted to sinus rhythm $(p<0.05$, compared with ibutilide). The mean rate of $\mathrm{AF}$ was 126 (26) $\mathrm{bpm}$ ( $\mathrm{p}=\mathrm{ns}$, compared with ibutilide) and the mean $\mathrm{QT}_{\mathrm{c}}$ interval in those who converted was 377 (28) ms $(p<0.05$, compared with ibutilide). One patient developed severe bradycardia. Ibutilide is effective for conversion of recent onset $A F$ in patients presenting to the $E D$ and there is a low rate of complications from ibutilide in this setting.

A trial fibrillation $(\mathrm{AF})$ is an arrhythmia commonly encountered in the emergency department (ED). Patients come to the ED with different clinical presentations of $\mathrm{AF}$ and the best treatment strategy varies, depending on number of factors. Among those who have a recent onset $(<48$ hours) of $\mathrm{AF}$ the most advantageous strategy may be restoration of sinus rhythm. ${ }^{1}$

Ibutilide is a class III antiarrhythmic drug which is only available in the intravenous form. It is approved for conversion of recent onset AF and atrial flutter. ${ }^{2}$ As is the case with other class III antiarrhythmic drugs ibutilide increases the risk of prolongation of the QT interval, ${ }^{2}$ which can predispose the patient to torsades de pointes. The risk of torsades de pointes with ibutilide is approximately $7-8 \%$ with $2-3 \%$ being sustained. ${ }^{2}$

We undertook this study to compare ibutilide with the strategy of giving rate control drugs only to those patients who presented to the ED with recent onset $\mathrm{AF}$ ( $<7$ days) and were in the non-fasting state, and therefore not candidates for immediate electrical cardioversion. We also aimed to assess the safety of ibutilide administration in the ED setting.

\section{METHODS}

\section{Study design}

We performed a retrospective analysis of all patients who had received ibutilide for AF in the ED between 2000 and 2003. For comparison we included a group of patients with recent onset $\mathrm{AF}$ treated with rate control drugs. The choice of treatment for each patient was at the discretion of the attending physician.

\section{Study setting, population, and drug dosing}

The Icelandic Data Protection Authority and the Landspitali University Hospital Institutional Review Board gave permission for the undertaking of this study. As this was a retrospective study, the Institutional Review Board did not require written informed consent from the participants.

This study was undertaken in the university hospital's Hringbraut campus emergency room where cardiac patients are treated. In our ED there are strict exclusion criteria for the use of ibutilide, which were set when ibutilide use began. Those patients with a decreased ejection fraction of the left ventricle, a history of long QT interval, AF with slow ventricular response $(<65 \mathrm{bpm})$, or electrolyte disturbances, or those taking other QT prolonging drugs are excluded from receiving the drug. This is to minimise the risk of serious adverse effects of the drug, especially torsades de pointes. ${ }^{3}$ Ibutilide dosing is as follows: $1 \mathrm{mg}$ given intravenously over 10 minutes followed by a 10 minute interval before another $1 \mathrm{mg}$ dose given if AF had not converted by that time. Ibutilide administration is stopped immediately if conversion to sinus rhythm has occurred.

\section{Data analysis}

Student's $t$ test was used for comparison between groups. A $\mathrm{p}$ value $<0.05$ was considered significant. Data are presented as mean $(\mathrm{SD})$.

\section{RESULTS}

\section{Ibutilide group}

A total of 22 patients (mean (SD) age 55 (13) years; 20 men) with AF received ibutilide. The mean rate of the AF was 137 (24) bpm. In all patients the duration of symptoms was less than seven days. Ten patients received two doses of $1 \mathrm{mg}$ each whereas 12 required only $1 \mathrm{mg}$. Fourteen patients (64\%) converted to sinus rhythm, all within one hour of the initial infusion (fig 1). The mean $\mathrm{QT}_{\mathrm{C}}$ interval after conversion in the 14 patients was $420(28) \mathrm{ms}$ and there were no instances of torsades de pointes or other complications. All were discharged from the ED without incidence after four hours.

\section{Rate control group}

A total of 24 patients (mean age 71 (12) years; 11 men) with AF received no membrane active antiarrhythmics but only rate control drugs. Of these, eight received a $\beta$ blocker only, four received calcium channel blockers, and nine received digoxin. Two patients were given both a $\beta$ blocker and digoxin and one received both a $\beta$ blocker and a calcium channel blocker. In all patients the duration of the arrhythmia was less than seven days. The mean rate of AF was 126 (26) bpm ( $p=$ not significant compared with ibutilide). Seven of the 24 patients $(29 \%)$ converted to sinus rhythm within 12 hours $(\mathrm{p}<0.005$, compared with conversion rate at one hour with ibutilide). The mean $\mathrm{QT}_{\mathrm{c}}$ interval in those who converted was 377 (28) ms $(\mathrm{p}<0.05$, compared 


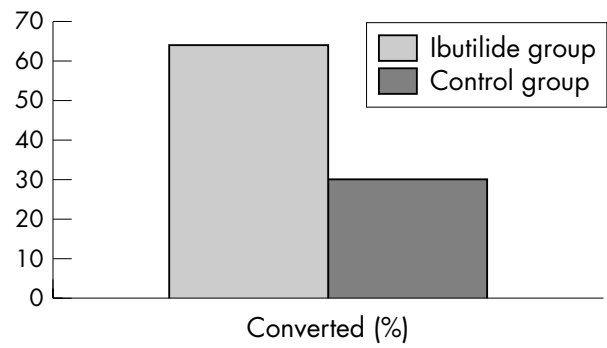

Figure 1 Rate of conversion to sinus rhythm with ibutilide versus rate control drugs.

to ibutilide) and there was one incidence of severe bradycardia following administration of digoxin.

\section{DISCUSSION}

The main results of our study suggest that ibutilide is more successful than rate control drugs in early restoration of sinus rhythm in recent onset AF. Ibutilide appears to be a safe drug to use in the ED setting.

$\mathrm{AF}$ is probably the commonest arrhythmia in the ED. Many patients will have had AF for less than 48 hours and are therefore candidates for cardioversion without preceding anticoagulation. Early restoration of sinus rhythm in this group of patients is important and feasible. ${ }^{1}$ Electrical cardioversion is frequently used to restore sinus rhythm but requires that patients are fasting. There is also an added cost of anaesthesia. Pharmacological cardioversion, however, may be attempted even if the patient is non-fasting.

A number of drugs have been found effective for rapid cardioversion of AF. A recently published meta-analysis concluded, albeit from a limited number of comparative studies, that flecainide was superior to many of the other antiarrhythmic drugs in this respect. ${ }^{4}$ Flecainide has a conversion rate of $57-68 \%$ at two to four hours after drug administration. ${ }^{5}$ Ibutilide is a recent addition to the armamentarium of drugs for pharmacological cardioversion of AF and has been shown to be superior to both procainamide and sotalol for this purpose. ${ }^{67}$

The rate of success of ibutilide in our study was $64 \%$ in the first hour after administration of the first dose which is comparable with what is reported with flecainide. A recently published study comparing ibutilide and flecainide found their efficacy to be comparable. ${ }^{8}$ As a result of strict exclusion criteria for ibutilide administration no complications were seen in the this group. Patients were observed in a monitor for four hours after administration of ibutilide and then discharged, with no problems reported after discharge.
There are several limitations to this study. The data collection was done retrospectively and therefore the patients were not randomised. The groups are thus not identical and the patients in the rate control group were older than those in the ibutilide group. Consequently, patients in the rate control group may conceivably have had more comorbidities.

\section{Conclusion}

Ibutilide is effective for converting recent onset AF in patients presenting to the ED and is an alternative for cardioversion in those patients who are non-fasting and therefore are not candidates for immediate electrical cardioversion. Ibutilide has a rapid mode of action with a high rate of conversion in the first hour after drug administration. The main potential drawback of the drug is the risk of torsades de pointes. However, as our study shows, the incidence of complications is low in selected patients and the drug can be safely used in the ED setting.

\section{Authors' affiliations}

O Viktorsdottir, A Henriksdottir, D O Arnar, Department of Emergency Medicine, Landspitali University Hospital, Reykjavik, Iceland

Competing interests: none declared

Correspondence to: Dr D O Arnar, Department of Emergency Medicine, Landspitali University Hospital, Hringbraut, 101 Reykjavik; davidar@ landspitali.is

Accepted for publication 8 March 2005

\section{REFERENCES}

1 Jacoby JL, Cesta M, Heller MB, et al. Synchronized emergency department cardioversion of atrial dysrhythmias saves time, money and resources. J Emerg Med 2005;28:27-30

2 Stambler BS, Wood MA, Ellenbogen KA, et al. Efficacy and safety of repeated intravenous doses of ibutilide for rapid conversion of atrial fibrillation and flutter. Circulation 1996;94:1613-21.

3 Locati EH, Maison-Blanche P, Dejode P, et al. Spontaneous sequences of onset of torsade de pointes in patients with aquired prolonged repolarisation: Quantitative analysis of Holter recordings. J Am Coll Cardiol 1995;25:1564-75.

4 McNamara RL, Tamariz $\sqcup$, Segal JS, et al. Management of atrial fibrillation: review of the evidence for the role of pharmacologic therapy, electrical cardioversion, and echocardiography. Ann Intern Med 2003;139:1018-33.

5 Khan IA. Oral loading single dose flecainide for pharmacologic cardioversion of recent onset atrial fibrillation. Int J Cardiol 2003;87:121-8.

6 Stambler BS, Wood MA, Ellenbeogen KA. Antiarrhythmic actions of intravenous ibutilide compared with procainamide during human atrial flutter and fibrillation: electrophysiological determinants of enhanced conversion efficacy. Circulation 1997;96:4298-306.

7 Vos MA, Golitsyn SR, Stangl K, et al. Superiority of ibutilide (a new class III agent) over $D, L$ sotalol in converting atrial flutter and fibrillation. The ibutilide/ sotalol comparator group. Heart 1998;79:568-75.

8 Reisinger J, Gatterer E, Lang W, et al. Flecainide versus ibutilide for immediate cardioversion of atrial fibrillation of recent onset. Eur Heart $J$ 2004;25:1318-24. 